\begin{tabular}{ll} 
C O L L O Q U I U M & M A T H E M A T I C U M \\
\hline vol. LXV & 1993 \\
\hline
\end{tabular}

\title{
ON AN EXTENDED CONTACT BOCHNER CURVATURE TENSOR ON CONTACT METRIC MANIFOLDS
}

BY

\author{
HIROSHI ENDO (ICHIKAWA)
}

1. Introduction. On Sasakian manifolds, Matsumoto and Chūman [3] defined a contact Bochner curvature tensor (see also Yano [7]) which is invariant under $D$-homothetic deformations (for $D$-homothetic deformations, see Tanno [5]). On the other hand, Tricerri and Vanhecke [6] defined a general Bochner curvature tensor with conformal invariance on almost Hermitian manifolds.

In this paper we define an extended contact Bochner curvature tensor which is invariant under $D$-homothetic deformations of contact metric manifolds; we call it the EK-contact Bochner curvature tensor. We show that a contact metric manifold with vanishing EK-contact Bochner curvature tensor is a Sasakian manifold.

2. Preliminaries. Let $M$ be a $(2 n+1)$-dimensional contact metric manifold with structure tensors $(\phi, \xi, \eta, g)$. They satisfy

$$
\begin{gathered}
\phi \xi=0, \quad \eta(\xi)=1, \quad \phi^{2}=-I+\eta \otimes \xi, \quad \eta(X)=g(\xi, X), \\
g(\phi X, \phi Y)=g(X, Y)-\eta(X) \eta(Y), \quad g(\phi X, Y)=d \eta(X, Y)
\end{gathered}
$$

for any vector fields $X$ and $Y$ on $M$. Define an operator $h$ by $h=-\frac{1}{2} \mathcal{L}_{\xi} \phi$, where $\mathcal{L}$ denotes Lie differentiation. Then the vector field $\xi$ is Killing if and only if $h$ vanishes. It is well known that $h$ and $\phi h$ are symmetric operators, $h$ anti-commutes with $\phi$ (i.e., $\phi h+h \phi=0), h \xi=0, \eta \circ h=0, \operatorname{Tr} h=0$ and $\operatorname{Tr} \phi h=0$. Moreover, for every contact metric manifold $M$ the following general formulas were obtained:

$$
\begin{gathered}
\nabla_{X} \xi=\phi X+\phi h X \\
\frac{1}{2}(R(\xi, X) \xi-\phi R(\xi, \phi X) \xi)=h^{2} X+\phi^{2} X \\
g(Q \xi, \xi)=2 n-\operatorname{Tr} h^{2}, \\
\sum_{i=1}^{2 n+1}\left(\nabla_{E_{i}} \phi\right) E_{i}=-2 n \xi \quad\left(\left\{E_{i}\right\} \text { is an orthonormal frame }\right),
\end{gathered}
$$


(2.5) $\left(\nabla_{\phi X} \phi\right) \phi Y+\left(\nabla_{X} \phi\right) Y=-2 g(X, Y) \xi+\eta(Y)(X+h X+\eta(X) \xi)$,

$$
\phi\left(\nabla_{\xi} h\right) X=X-\eta(X) \xi-h^{2} X-R(X, \xi) \xi,
$$

where $\nabla$ is the covariant differentiation with respect to $g, Q$ is the Ricci operator of $M, R$ is the curvature tensor field of $M$ and $\operatorname{Tr} h$ denotes the trace of $h$ (cf. [1], [2] and [4]). Moreover, using $\phi h \xi=0$, we get

$$
\phi\left(\nabla_{Y} h\right) \xi=-h Y-h^{2} Y .
$$

If $\xi$ is Killing on a contact metric manifold $M$, then $M$ is said to be a $K$-contact Riemannian manifold. If a contact metric manifold $M$ is normal (i.e., $N+2 d \eta \otimes \xi=0$, where $N$ denotes the Nijenhuis tensor formed with $\phi$ ), then $M$ is called a Sasakian manifold. Every Sasakian manifold is a K-contact Riemannian manifold. On a Sasakian manifold with structure tensors $(\phi, \xi, \eta, g)$, we have

$$
\begin{gathered}
\nabla_{X} \xi=\phi X, \quad\left(\nabla_{X} \phi\right) Y=R(X, \xi) Y=-g(X, Y) \xi+\eta(Y) X, \\
\phi Q=Q \phi, \quad Q \xi=2 n \xi
\end{gathered}
$$

(see, e.g., [8]).

3. $D$-homothetic deformations. Let $M$ be an $(m+1)$-dimensional $(m=2 n)$ contact metric manifold. Define the tensor field $B^{\text {es }}$ on $M$ by

$$
\begin{aligned}
& B^{\mathrm{es}}(X, Y) \\
= & R(X, Y)+h \phi X \wedge h \phi Y+\frac{1}{2(m+4)}(Q Y \wedge X-(\phi Q \phi Y) \wedge X \\
& +\frac{1}{2}(\eta(Y) Q \xi \wedge X+\eta(Q Y) \xi \wedge X)-Q X \wedge Y+(\phi Q \phi X) \wedge Y \\
& -\frac{1}{2}(\eta(X) Q \xi \wedge Y+\eta(Q X) \xi \wedge Y)+(Q \phi Y) \wedge \phi X+(\phi Q Y) \wedge \phi X \\
& -(Q \phi X) \wedge \phi Y-(\phi Q X) \wedge \phi Y+2 g(Q \phi X, Y) \phi+2 g(\phi Q X, Y) \phi \\
& +2 g(\phi X, Y) \phi Q+2 g(\phi X, Y) Q \phi-\eta(X) Q Y \wedge \xi+\eta(X)(\phi Q \phi Y) \wedge \xi \\
& +\eta(Y) Q X \wedge \xi-\eta(Y)(\phi Q \phi X) \wedge \xi) \\
& -\frac{k+m}{m+4}(\phi Y \wedge \phi X+2 g(\phi X, Y) \phi)-\frac{k-4}{m+4} Y \wedge X \\
& +\frac{k}{m+4}(\eta(Y) \xi \wedge X+\eta(X) Y \wedge \xi) \\
& -\frac{1}{(m+4)(m+2)} \operatorname{Tr} h^{2}(\phi Y \wedge \phi X \\
& +2 g(\phi X, Y) \phi+Y \wedge X+\eta(X) \xi \wedge Y-\eta(Y) \xi \wedge X),
\end{aligned}
$$

where $k=\frac{S+m}{m+2}(S$ is the scalar curvature tensor of $M)$ and $(X \wedge Y) Z=$ 
$g(Y, Z) X-g(X, Z) Y$ (cf. [3]). (3.1) implies the following identities:

$$
\begin{aligned}
& B^{\mathrm{es}}(X, Y) Z=-B^{\mathrm{es}}(Y, X) Z, \\
& B^{\mathrm{es}}(X, Y) Z+B^{\mathrm{es}}(Y, Z) X+B^{\mathrm{es}}(Z, X) Y=0, \\
& g\left(B^{\mathrm{es}}(X, Y) Z, W\right)=-g\left(Z, B^{\mathrm{es}}(X, Y) W\right), \\
& g\left(B^{\mathrm{es}}(X, Y) Z, W\right)=g\left(B^{\mathrm{es}}(Z, W) X, Y\right) .
\end{aligned}
$$

If $M$ is a Sasakian manifold, then $B^{\text {es }}$ coincides with the contact Bochner curvature tensor of Matsumoto and Chüman [3] and

$$
B^{\mathrm{es}}(\xi, Y) Z=B^{\mathrm{es}}(X, Y) \xi=0, \quad B^{\mathrm{es}}(\phi X, \phi Y) Z=B^{\mathrm{es}}(X, Y) Z,
$$

where we have used $R(\phi X, Y) Z-R(\phi Y, X) Z=g(\phi Z, X) Y-g(\phi Z, Y) X-$ $g(Z, X) \phi Y+g(Z, Y) \phi X$ on a Sasakian manifold.

Consider a D-homothetic deformation $g^{*}=\alpha g+\alpha(\alpha-1) \eta \otimes \eta, \phi^{*}=$ $\phi, \xi^{*}=\alpha^{-1} \xi, \eta^{*}=\alpha \eta$ on a contact metric manifold $M$, where $\alpha$ is a positive constant. We then say that $M(\phi, \xi, \eta, g)$ is D-homothetic to $M\left(\phi^{*}, \xi^{*}, \eta^{*}, g^{*}\right)$. It is well known that if a contact metric manifold $M(\phi, \xi, \eta, g)$ is $D$-homothetic to $M\left(\phi^{*}, \xi^{*}, \eta^{*}, g^{*}\right)$, then $M\left(\phi^{*}, \xi^{*}, \eta^{*}, g^{*}\right)$ is a contact metric manifold. Moreover, if $M(\phi, \xi, \eta, g)$ is a K-contact Riemannian manifold (resp. Sasakian manifold), then so is $M\left(\phi^{*}, \xi^{*}, \eta^{*}, g^{*}\right)$ (see [5]). Denoting by $W^{i}{ }_{j k}$ the difference $\stackrel{*}{i}_{j k}-\Gamma^{i}{ }_{j k}$ of Christoffel symbols, by (2.1) on a contact metric manifold $M$ we have

$$
\begin{aligned}
W(X, Y)= & (\alpha-1)(\eta(Y) \phi X+\eta(X) \phi Y) \\
& +\frac{\alpha-1}{2 \alpha}\left(\left(\nabla_{X} \eta\right)(Y)+\left(\nabla_{Y} \eta\right)(X)\right) \xi \quad(\text { see }[5]) \\
= & (\alpha-1)(\eta(Y) \phi X+\eta(X) \phi Y)+\frac{\alpha-1}{\alpha} g(\phi h X, Y) \xi .
\end{aligned}
$$

Putting this into

$$
\begin{aligned}
R^{*}(X, Y) Z= & R(X, Y) Z+\left(\nabla_{X} W\right)(Z, Y)-\left(\nabla_{Y} W\right)(Z, X) \\
& +W(W(Z, Y), X)-W(W(Z, X), Y)
\end{aligned}
$$

and using (2.1) we have

$$
\begin{aligned}
R^{*}(X, Y) Z= & R(X, Y) Z+(\alpha-1)(2 g(\phi X, Y) \phi Z+g(\phi Z, Y) \phi X \\
& -g(\phi Z, X) \phi Y+\eta(Y)\left(\nabla_{X} \phi\right)(Z)+\eta(Z)\left(\nabla_{X} \phi\right)(Y) \\
& \left.-\eta(X)\left(\nabla_{Y} \phi\right)(Z)-\eta(Z)\left(\nabla_{Y} \phi\right)(X)\right) \\
& -(\alpha-1)^{2}(\eta(Z) \eta(X) Y-\eta(Z) \eta(Y) X) \\
& -\frac{\alpha-1}{\alpha}\left(g\left(X,\left(\nabla_{Y} \phi\right) h Z\right) \xi-g\left(Y,\left(\nabla_{X} \phi\right) h Z\right) \xi\right. \\
& +g\left(X, \phi\left(\nabla_{Y} h\right) Z\right) \xi-g\left(Y, \phi\left(\nabla_{X} h\right) Z\right) \xi \\
& +g(X, \phi h Z) \phi h Y-g(Y, \phi h Z) \phi h X)
\end{aligned}
$$




$$
-\frac{(\alpha-1)^{2}}{\alpha}(\eta(X) g(h Z, Y) \xi-\eta(Y) g(h Z, X) \xi) .
$$

Choosing a $\phi^{*}$-basis with respect to $g^{*}$ and using (2.4) and (2.6), we get

(3.5) $\operatorname{Ric}^{*}(X, Y)$

$$
\begin{aligned}
= & \operatorname{Ric}(X, Y)+(\alpha-1)(-2 g(X, Y)+2(2 n+1) \eta(X) \eta(Y)) \\
& +2 n(\alpha-1)^{2} \eta(X) \eta(Y)-\frac{\alpha-1}{\alpha}(-g(X, Y)+\eta(X) \eta(Y) \\
& -2 g(h X, Y)+g(h X, h Y)+g(R(X, \xi) \xi, Y)),
\end{aligned}
$$

where Ric is the Ricci curvature of $M$.

From (3.5), we find

$$
\begin{aligned}
Q^{*} X= & \frac{1}{\alpha} Q X+\frac{\alpha-1}{\alpha}(-2 X+2(2 n+1) \eta(X) \xi) \\
& -\frac{\alpha-1}{\alpha^{2}} g(X, Q \xi) \xi-2 n\left(\frac{\alpha-1}{\alpha}\right)^{2} \eta(X) \xi \\
& -\frac{\alpha-1}{\alpha^{2}}\left(-X+\eta(X) \xi-2 h X+h^{2} X+R(X, \xi) \xi\right),
\end{aligned}
$$

where we have used the fact that

$$
Q^{*} \xi=\frac{1}{\alpha} Q \xi-\frac{\alpha-1}{\alpha^{2}} g(Q \xi, \xi) \xi+2 n \frac{\alpha^{2}-1}{\alpha^{2}} \xi .
$$

By virtue of (2.3) we have

$$
S^{*}=\frac{1}{\alpha} S-2 n \frac{\alpha-1}{\alpha}+\frac{\alpha-1}{\alpha^{2}} \operatorname{Tr} h^{2} .
$$

Moreover, using the definition of $h$, we have

$$
h^{*}=\frac{1}{\alpha} h,
$$

from which we get

$$
\operatorname{Tr} h^{* 2}=\frac{1}{\alpha^{2}} \operatorname{Tr} h^{2} .
$$

By means of (2.2), (3.1) and (3.4)-(3.9), after some lengthy computation, we obtain

$$
\begin{aligned}
& (3.10) \quad{ }^{B^{\operatorname{es}}}(X, Y) Z \\
& =B^{\operatorname{es}}(X, Y) Z+(\alpha-1)(\eta(Y) g(X, Z) \xi-\eta(X) g(Y, Z) \xi \\
& \quad+2 \eta(X) \eta(Z) Y-2 \eta(Y) \eta(Z) X+\eta(Y)\left(\nabla_{X} \phi\right) Z+\eta(Z)\left(\nabla_{X} \phi\right) Y \\
& \left.\quad-\eta(X)\left(\nabla_{Y} \phi\right) Z-\eta(Z)\left(\nabla_{Y} \phi\right) X\right) \\
& \quad-\frac{\alpha-1}{\alpha}\left(g\left(X,\left(\nabla_{Y} \phi\right) h Z\right) \xi-g\left(Y,\left(\nabla_{X} \phi\right) h Z\right) \xi\right.
\end{aligned}
$$




$$
\begin{aligned}
& \left.+g\left(X, \phi\left(\nabla_{Y} h\right) Z\right) \xi-g\left(Y, \phi\left(\nabla_{X} h\right) Z\right) \xi\right) \\
& -\frac{(\alpha-1)^{2}}{\alpha}(\eta(X) g(h Z, Y) \xi-\eta(Y) g(h Z, X) \xi) \\
& +\frac{1}{2(2 n+4)}\left\{\frac{3(\alpha-1)}{2 \alpha}(\eta(X) \eta(Z) g(Y, Q \xi) \xi-\eta(Y) \eta(Z) g(X, Q \xi) \xi\right. \\
& +g(Y, Z) g(X, Q \xi) \xi-g(X, Z) g(Y, Q \xi) \xi) \\
& +\frac{1}{2} \frac{\alpha-1}{\alpha}(g(Y, Z) \eta(X)-g(X, Z) \eta(Y)) g(Q \xi, \xi) \xi \\
& +\frac{\alpha-1}{\alpha}(-g(\phi X, Z) g(\phi Y, Q \xi) \xi+g(\phi Y, Z) g(\phi X, Q \xi) \xi \\
& -2 g(\phi X, Y) g(\phi Z, Q \xi) \xi+4 n g(X, Z) \eta(Y) \xi-4 n g(Y, Z) \eta(X) \xi)\}
\end{aligned}
$$

Now we introduce the EK-contact Bochner curvature tensor $B^{\mathrm{ek}}$ on $M$ by

$$
\begin{aligned}
B^{\mathrm{ek}}( & X, Y) Z \\
= & B^{\mathrm{es}}(X, Y) Z-\eta(X) B^{\mathrm{es}}(\xi, Y) Z-\eta(Y) B^{\mathrm{es}}(X, \xi) Z \\
& -\eta(Z) B^{\mathrm{es}}(X, Y) \xi-\eta\left(B^{\mathrm{es}}(X, Y) Z\right) \xi+\eta(X) \eta\left(B^{\mathrm{es}}(\xi, Y) Z\right) \xi \\
& +\eta(Y) \eta\left(B^{\mathrm{es}}(X, \xi) Z\right) \xi+\eta(Y) \eta(Z) \phi B^{\mathrm{es}}(\phi X, \xi) \xi \\
& +\eta(X) \eta(Z) \phi B^{\mathrm{es}}(\xi, \phi Y) \xi .
\end{aligned}
$$

In particular, if $M$ is a Sasakian manifold, then $B^{\mathrm{ek}}=B^{\mathrm{es}}$ from (3.2) and (3.3). That is, $B^{\text {ek }}$ coincides with the contact Bochner curvature tensor defined by Matsumoto and Chūman [3].

THEOREM 3.1. The EK-contact Bochner curvature tensor is invariant under D-homothetic deformations $M(\phi, \xi, \eta, g) \rightarrow M\left(\phi^{*}, \xi^{*}, \eta^{*}, g^{*}\right)$ on a contact metric manifold $M$.

Proof. Using (2.5)-(2.7) and (3.10), we find

$$
\begin{aligned}
-\eta^{*}(X) & \stackrel{*}{B^{\mathrm{es}}}\left(\xi^{*}, Y\right) Z=-\eta(X) \stackrel{*}{B^{\mathrm{es}}}(\xi, Y) Z \\
= & -\eta(X) B^{\mathrm{es}}(\xi, Y) Z \\
& +(\alpha-1)\left(-\eta(X) \eta(Z) Y+\eta(X) \eta(Z) h Y+\eta(X)\left(\nabla_{Y} \phi\right) Z\right) \\
& +\frac{\alpha-1}{\alpha}(\eta(X) \eta(Y) \eta(Z) \xi+\eta(X) g(Y, R(Z, \xi) \xi) \xi) \\
& +\frac{(\alpha-1)^{2}}{\alpha} \eta(X) g(Y, Z) \xi
\end{aligned}
$$




$$
\begin{aligned}
& +\frac{(\alpha-1)(\alpha-2)}{\alpha} \eta(X) g(Y, h Z) \xi \\
& +\frac{1}{2(2 n+4)}\left\{\frac{3(\alpha-1)}{2 \alpha}(-g(Y, Z) \eta(X) g(Q \xi, \xi) \xi\right. \\
& +\eta(X) \eta(Y) \eta(Z) g(Q \xi, \xi) \xi) \\
& +\frac{1}{2} \frac{\alpha-1}{\alpha}(-\eta(X) g(Y, Z) g(Q \xi, \xi) \xi \\
& +\eta(X) \eta(Y) \eta(Z) g(Q \xi, \xi) \xi) \\
& \left.+\frac{\alpha-1}{\alpha}(4 n g(Y, Z) \eta(X) \xi-4 n \eta(X) \eta(Y) \eta(Z) \xi)\right\}
\end{aligned}
$$

(3.13) $\quad-\eta^{*}(Y) \stackrel{*}{B}^{\mathrm{es}}\left(X, \xi^{*}\right) Z$

$$
\begin{aligned}
= & -\eta(Y) B^{\mathrm{es}}(X, \xi) Z+(\alpha-1)(\eta(Y) \eta(Z) X \\
& \left.-\eta(Y) \eta(Z) h X-\eta(Y)\left(\nabla_{X} \phi\right) Z\right) \\
& -\frac{\alpha-1}{\alpha}(\eta(X) \eta(Y) \eta(Z) \xi+\eta(Y) g(X, R(Z, \xi) \xi) \xi) \\
& -\frac{(\alpha-1)^{2}}{\alpha} g(X, Z) \eta(Y) \xi \\
& -\frac{(\alpha-1)(\alpha-2)}{\alpha} g(h X, Z) \eta(Y) \xi \\
& +\frac{1}{2(2 n+4)}\left\{\frac{3(\alpha-1)}{2 \alpha}(g(X, Z) \eta(Y) g(Q \xi, \xi) \xi\right. \\
& -\eta(X) \eta(Y) \eta(Z) g(Q \xi, \xi) \xi) \\
& +\frac{1}{2} \frac{\alpha-1}{\alpha}(g(X, Z) \eta(Y) g(Q \xi, \xi) \xi-\eta(X) \eta(Y) \eta(Z) g(Q \xi, \xi) \xi) \\
& \left.+\frac{\alpha-1}{\alpha}(-4 n g(X, Z) \eta(Y) \xi+4 n \eta(X) \eta(Y) \eta(Z) \xi)\right\},
\end{aligned}
$$

(3.14) $\quad-\eta^{*}(Z) B^{* \mathrm{es}}(X, Y) \xi^{*}$

$$
\begin{aligned}
= & -\eta(Z) B^{\mathrm{es}}(X, Y) \xi+(\alpha-1)(-\eta(X) \eta(Z) Y+\eta(Y) \eta(Z) X \\
& -\eta(Y) \eta(Z) h X+\eta(X) \eta(Z) h Y-\eta(Z)\left(\nabla_{X} \phi\right) Y \\
& \left.+\eta(Z)\left(\nabla_{Y} \phi\right) X\right),
\end{aligned}
$$

(3.15) $-\eta^{*}\left({ }^{*}\right.$ es $\left.(X, Y) Z\right) \xi^{*}=-\eta\left(B^{\mathrm{es}}(X, Y) Z\right) \xi-\frac{\alpha-1}{\alpha}(\eta(X) g(h Y, Z) \xi$ $-\eta(Y) g(h X, Z) \xi-g\left(X,\left(\nabla_{Y} \phi\right) h Z\right) \xi+g\left(Y,\left(\nabla_{X} \phi\right) h Z\right) \xi$ 


$$
\begin{aligned}
& \left.-g\left(X, \phi\left(\nabla_{Y} h\right) Z\right) \xi+g\left(Y, \phi\left(\nabla_{X} h\right) Z\right) \xi\right) \\
& +\frac{1}{2(2 n+4)}\left\{\frac{3(\alpha-1)}{2 \alpha}(-g(Y, Z) g(X, Q \xi) \xi\right. \\
& +g(X, Z) g(Y, Q \xi) \xi-\eta(X) \eta(Z) g(Y, Q \xi) \xi \\
& +\eta(Y) \eta(Z) g(X, Q \xi) \xi)+\frac{1}{2} \frac{\alpha-1}{\alpha}(-\eta(X) g(Y, Z) g(Q \xi, \xi) \xi \\
& +\eta(Y) g(X, Z) g(Q \xi, \xi) \xi)+\frac{\alpha-1}{\alpha}(g(\phi X, Z) g(\phi Y, Q \xi) \xi \\
& -g(\phi Y, Z) g(\phi X, Q \xi) \xi+2 g(\phi X, Y) g(\phi Z, Q \xi) \xi \\
& -4 n g(X, Z) \eta(Y) \xi+4 n g(Y, Z) \eta(X) \xi)\} .
\end{aligned}
$$

Using (2.3), (2.5), (2.6) and (3.15), we get

$$
\begin{aligned}
& \eta^{*}(X) \eta^{*}\left(\stackrel{*}{B}^{\mathrm{es}}\left(\xi^{*}, Y\right) Z\right) \xi^{*}+\eta^{*}(Y) \eta^{*}\left(\stackrel{*}{B}^{\mathrm{es}}\left(X, \xi^{*}\right) Z\right) \xi^{*} \\
&= \frac{\alpha-1}{\alpha}(2 g(Y, h Z) \eta(X) \xi-2 g(X, h Z) \eta(Y) \xi+g(Y, Z) \eta(X) \xi \\
&\quad-g(X, Z) \eta(Y) \xi-g(Y, R(Z, \xi) \xi) \eta(X) \xi+g(X, R(Z, \xi) \xi) \eta(Y) \xi) \\
&+\frac{1}{2 n+4} \frac{\alpha-1}{\alpha}(g(X, Z) \eta(Y) \xi-g(Y, Z) \eta(X) \xi) \operatorname{Tr} h^{2} .
\end{aligned}
$$

From (2.5) and (3.14) we have

$$
\begin{aligned}
\eta^{*}(Y) \eta^{*}(Z) \phi^{*} B^{\mathrm{es}}\left(\phi^{*} X, \xi^{*}\right) \xi^{*}= & \eta(Y) \eta(Z) \phi B^{\mathrm{es}}(\phi X, \xi) \xi \\
& +2(\alpha-1) \eta(Y) \eta(Z) h X, \\
\eta^{*}(X) \eta^{*}(Z) \phi^{*} B^{\mathrm{es}}\left(\xi^{*}, \phi^{*} Y\right) \xi^{*}= & -\eta(X) \eta(Z) \phi B^{\mathrm{es}}(\xi, \phi Y) \xi \\
& -2(\alpha-1) \eta(X) \eta(Z) h Y .
\end{aligned}
$$

Therefore, using (2.3) and (3.10)-(3.17), we get our result.

4. Contact metric manifolds with vanishing EK-contact Bochner curvature tensor. We define

$$
s^{\#}=\sum_{i, j=1}^{2 n+1} g\left(R\left(E_{i}, E_{j}\right) \phi E_{j}, \phi E_{i}\right),
$$

where $\left\{E_{i}\right\}$ is an orthonormal frame.

Lemma $4.1([4])$. For any $(2 n+1)$-dimensional contact metric manifold $M$ we have

$$
s^{\#}-S+4 n^{2}=\operatorname{Tr} h^{2}+\frac{1}{2}\left\{\|\nabla \phi\|^{2}-4 n\right\} \geq 0 .
$$


Moreover, $M$ is Sasakian if and only if $\|\nabla \phi\|^{2}-4 n=0$ or equivalently

$$
s^{\#}-S+4 n^{2}=0 .
$$

THEOREM 4.1. Let $M$ be a contact metric manifold with vanishing EKcontact Bochner curvature tensor. Then $M$ is a Sasakian manifold.

Proof. Since the EK-contact Bochner curvature tensor of $M$ vanishes we have

$$
\begin{aligned}
g\left(B^{\mathrm{es}}(X, Y) Z\right. & , W) \\
= & \eta(X) g\left(B^{\mathrm{es}}(\xi, Y) Z, W\right)+\eta(Y) g\left(B^{\mathrm{es}}(X, \xi) Z, W\right) \\
& +\eta(Z) g\left(B^{\mathrm{es}}(X, Y) \xi, W\right)+\eta(W) \eta\left(B^{\mathrm{es}}(X, Y) Z\right) \\
& -\eta(X) \eta(W) \eta\left(B^{\mathrm{es}}(\xi, Y) Z\right)-\eta(Y) \eta(W) \eta\left(B^{\mathrm{es}}(X, \xi) Z\right) \\
& -\eta(Y) \eta(Z) g\left(\phi B^{\mathrm{es}}(\phi X, \xi) \xi, W\right) \\
& -\eta(X) \eta(Z) g\left(\phi B^{\mathrm{es}}(\xi, \phi Y) \xi, W\right) .
\end{aligned}
$$

Setting $X=E_{i}, Y=E_{j}, Z=\phi E_{j}, W=\phi E_{i}\left(\left\{E_{i}\right\}\right.$ is a $\phi$-basis $)$ in each member of (4.2) and summing over $i$ and $j$, we have

$$
g\left(B^{\mathrm{es}}\left(E_{i}, E_{j}\right) \phi E_{j}, \phi E_{i}\right)=s^{\#}-S+4 n^{2}-2 \operatorname{Tr} h^{2}+\left(\operatorname{Tr} h^{2}\right)^{2}=0 .
$$

Using Lemma 4.1, we obtain

$$
\left(\operatorname{Tr} h^{2}\right)^{2}-\operatorname{Tr} h^{2}+\frac{1}{2}\left\{\|\nabla \phi\|^{2}-4 n\right\}=0 .
$$

On the other hand, from (4.2) we find

$$
\sum_{i=1}^{2 n+1} g\left(B^{\mathrm{es}}\left(E_{i}, \xi\right) \xi, E_{i}\right)=-\sum_{i=1}^{2 n+1} g\left(B^{\mathrm{es}}\left(\phi E_{i}, \xi\right) \xi, \phi E_{i}\right) .
$$

Hence $g(Q \xi, \xi)=2 n$. Thus we get our result.

\section{REFERENCES}

[1] D. E. Blair, Contact Manifolds in Riemannian Geometry, Lecture Notes in Math. 509, Springer, Berlin 1976.

[2] -, Critical associated metrics on contact manifolds, J. Austral. Math. Soc. 37 (1984), $82-88$.

[3] M. Matsumoto and G. Chūman, On the C-Bochner tensor, TRU Math. 5 (1969), $21-31$.

[4] Z. Olszak, On contact metric manifolds, Tôhoku Math. J. 31 (1979), 247-253.

[5] S. Tanno, The topology of contact Riemannian manifolds, Illinois J. Math. 12 (1968), $700-712$.

[6] F. Tricerri and L. Vanhecke, Curvature tensors on almost Hermitian manifolds, Trans. Amer. Math. Soc. 267 (1981), 365-398.

[7] K. Yano, Anti-invariant submanifolds of a Sasakian manifold with vanishing contact Bochner curvature tensor, J. Differential Geom. 12 (1977), 153-170. 
[8] K. Yano and M. Kon, Structures on Manifolds, World Sci., Singapore 1984.

KOHNODAI SENIOR HIGH SCHOOL

2-4-1, KOHNODAI

ICHIKAWA-SHI

CHIBA-KEN, 272

JAPAN

Reçu par la Rédaction le 24.6.1992 